\title{
Sulcoflex Piggyback Intraocular Lens Implantation For Correction Of Refractive Errors Following Cataract Surgery
}

\author{
Mona Purba, Associate Optometrist, Calgary, Alberta \\ Cynthia Mardinger BHSc, Cumming School of Medicine, University of Calgary \\ Abby Hibma BSc, Loma Linda University School of Medicine \\ Dr. Howard V Gimbel, MD, MPH, Clinical Professor University of Calgary, Clinical Professor Loma Linda University \\ Correspondence may be directed to: mkpurba@yahoo.ca
}

\begin{abstract}
Purpose: To investigate the visual and refractive outcomes following implantation of a Sulcoflex intraocular lens (IOL) to correct pseudophakic refractive error.

Methods: This retrospective chart review included 14 pseudophakic eyes of 13 patients who underwent implantation of a Sulcoflex IOL. The Sulcoflex IOL is a piggyback IOL technique where a secondary, supplementary lens is implanted in the ciliary sulcus.

Results: Eleven eyes (78.6\%) had a postoperative SE within $0.50 \mathrm{D}$ of the targeted SE. Preoperative UDVA was $20 / 30$ in 4 eyes $(28.6 \%$ ), and $20 / 40$ or worse in 10 eyes $(71.4 \%)$. Postoperative UDVA was 20/30 or better in all eyes, where half $(50.0 \%)$ of eyes were $20 / 20$ or better. The mean preoperative logMAR $(0.50 \pm$ 0.33 ) significantly improved to $0.06 \pm 0.09, p<.01$. There were no complications.

Conclusions. The significant improvement in UDVA and the precision in reaching the target refraction suggest that the Sulcoflex is a viable and successful treatment option for pseudophakic refractive error.
\end{abstract}

\section{Introduction}

Although today's patients expect exact refractive results following cataract surgery, more than minimal residual refractive errors still occur in some cases. ${ }^{1}$ Current surgical treatment options for correction of pseudophakic residual refractive error include: intraocular exchange (IOL) exchange, laser refractive surgery, and implantation of a piggyback IOL. ${ }^{1,2,3}$ The piggyback technique has classically consisted of the implantation of a secondary, supplementary IOL in the sulcus or in the capsular bag anterior to the primary IOL.,5 Recently, piggyback IOLs, such as the Sulcoflex (Rayner Intraocular Lenses Ltd.), have been specifically designed for implantation in the ciliary sulcus.

Removing the primary IOL can increase the risk of incurring capsular tears and zonular damage, IOL exchange is a procedure that is best performed during the early postoperative period before capsular bag fibrosis and adhesions occur., ${ }^{2,3}$ Unlike IOL exchange, the piggyback technique can be safely performed at any time after cataract surgery. ${ }^{6,7}$ Piggyback IOLs also show good predictability and accuracy because the IOL power can be calculated without knowing the cause of the residual refractive error. ${ }^{1,2}$ Although laser refractive surgery is commonly used to enhance pseudophakic refraction, it is best performed in patients with normal corneal topography and a stable refractive error. ${ }^{8}$ Piggyback IOLs are an excellent treatment option for patients who are not suitable candidates for laser treatment, such as those with high refractive error, a history of radial or astigmatic keratotomy, as well as those with corneal pathologies not amenable to refractive surgery. ${ }^{8,9}$ The piggyback lens is also seen as an attractive alternative to laser treatment, as it is reversible and avoids the significant corneal healing that follows excimer laser ablation. ${ }^{1,9}$ 
The Sulcoflex lens was the first anterior chamber piggyback IOL commercially available for the correction of both spherical and astigmatic refractive errors, and can provide patients with near or distance vision. ${ }^{4,10}$ In addition, Sulcoflex zonal refractive multifocal IOLs are available for the correction of presbyopia so as to provide patients freedom from spectacles. ${ }^{11,12}$ The purpose of this study was to investigate the safety and visual and refractive outcomes following implantation of the Sulcoflex lens to correct pseudophakic residual refractive error after routine cataract surgery.

\section{Methods}

\section{Study Design and Sample Recruitment}

This retrospective case series investigated the outcomes of patients who received implantation of a Sulcoflex IOL to correct residual refractive error following cataract surgery. This study included 14 pseudophakic eyes of 13 patients that received implantation of either a Sulcoflex aspheric IOL (Rayner 653L) or a Sulcoflex toric IOL (Rayner 653T) in the ciliary sulcus between October 2011 and June 2014 at the Gimbel Eye Centre in Calgary, Alberta. Patients with previous radial or astigmatic keratotomy were excluded due to possible diurnal visual fluctuations affecting the refractive outcome assessment. Patients who received implantation of Sulcoflex Multifocal IOLs were also excluded, as the scope of this study was limited to refractive outcome only. Data including target refraction, preoperative refraction and uncorrected distance visual acuity (UDVA) were collected. Postoperative refraction and UDVA data were collected at least 1 month after Sulcoflex implantation to ensure stability of visual outcomes. All participants provided informed consent for the surgical procedure, data collection and the establishment of a database.

\section{IOL Model Selection and Power Calculations}

The Sulcoflex is the first commercially available IOL specifically designed for secondary implantation in the ciliary sulcus. ${ }^{13}$ It is a one-piece hydrophilic acrylic IOL that is $14 \mathrm{~mm}$ in haptic diameter and $6.5 \mathrm{~mm}$ in optic diameter. ${ }^{14}$ The anterior convex and posterior concave lens configuration allow for adequate space between both IOLs, while its undulating haptics with $10^{\circ}$ of angulation allow for adequate uveal clearance. ${ }^{10,13}$ The Sulcoflex IOL is currently manufactured in aspheric, toric, and multifocal models. The Rayner 653L lens power is available from -10.00 to $+10.00 \mathrm{D}$, and the Rayner $653 \mathrm{~T}$ ranges from -7.00 to $+7.00 \mathrm{D}$ with cylinders between +1.00 to $+6.00 \mathrm{D} .{ }^{14}$ The Sulcoflex is not yet approved in Canada and was obtained with Health Canada approval. IOL types were selected based on the individual's refraction, lifestyle and needs. The IOL power and alignment was determined using Rayner's online IOL power calculator (Raytrace).

\section{Surgical Technique}

One surgeon, Dr. Howard Gimbel, performed all surgeries under topical and intracameral anesthetic. Eye-dependent incisions were made at variable meridians so as not to surgically induce or worsen pre-existing astigmatism. Sulcoflex IOLs were placed in the ciliary sulcus using the supplied injector. Good IOL centration was ensured. Toric IOLs were rotated into position by exact alignment of the reference marks on the toric IOL with corneal axis marks that were made prior to surgery. The wounds were watertight requiring no sutures. Patients were taken to the recovery room, and discharged when stable. Patients were instructed on postoperative ophthalmic medications.

\section{Statistical Analysis}

Mean, standard deviation, frequency, and percentage were used for descriptive statistics. For visual acuity evaluation, Snellen scale values were converted to $\log$ MAR notation. The Student paired t-test was used to compare preoperative and postoperative UDVA. The paired $t$-test was also used to compare the preoperative spectacle corrected distance visual acuity (SCDVA) with the postoperative UDVA, as a reference indicator to the efficacy of the Sulcoflex lens implantation. $P$-values less than 0.05 were considered statistically significant. Statistical analyses were conducted using SPSS version 21.

\section{Results}

This study included 7 women and 6 men of mean age 64 years (range 49 to 82 years). Table 1 shows patient demographics, as well as the type and power of the implanted Sulcoflex IOL. Of 8 aspheric and 6 toric IOLs, the mean IOL power was -0.36 $\mathrm{D} \pm 3.93$ (range -9.00 to $+5.00 \mathrm{D}$ ). The time between primary surgery and Sulcoflex implantation was less than 1 year in 8 eyes $(57.1 \%)$, between 1 and 5 years in 3 eyes $(21.4 \%)$, and over 5 years in 3 eyes $(21.4 \%)$, ranging from 7 months to 18 years. Postoperative follow-up visits ranged from 1.2 months to 14.6 months after surgery.

The mean spherical equivalent (SE) decreased from 0.16 $\mathrm{D} \pm 2.54$ (range -5.13 to $+3.00 \mathrm{D}$ ) preoperatively to -0.28 $\mathrm{D} \pm 0.60$ (range -1.00 to $+1.38 \mathrm{D}$ ) postoperatively. The mean target SE was $-0.15 \mathrm{D} \pm 0.21$ (range -0.50 to $0.00 \mathrm{D}$ ). The postoperative SE was within $0.25 \mathrm{D}$ of the targeted SE in 7 eyes (50.0\%), $0.50 \mathrm{D}$ in 11 eyes (78.6\%), $0.75 \mathrm{D}$ in 13 eyes (92.9\%), and had a greater than $1.00 \mathrm{D}$ difference in 1 eye (7.1\%). 
Table 1. Patient demographics and Sulcoflex intraocular lens (IOL) selection.

\begin{tabular}{|c|c|c|c|c|c|}
\hline Eye & Age (Y) & Gender & IOL Type & IOL Power (D) & $\begin{array}{c}\text { Time between primary and } \\
\text { secondary surgery (years) }\end{array}$ \\
\hline 1 & 52 & F & RAY653T & +0.50 & 0.85 \\
2 & 69 & M & RAY653L & +3.00 & 0.70 \\
3 & 65 & M & RAY653L & +2.50 & 0.81 \\
4 & 56 & F & RAY653L & +2.50 & 1.73 \\
5 & 68 & F & RAY653L & +3.00 & 0.76 \\
6 & 65 & M & RAY653L & +2.50 & 0.81 \\
7 & 73 & F & RAY653L & -2.00 & 1.30 \\
8 & 75 & F & RAY653L & -5.00 & 8.38 \\
9 & 49 & M & RAY653T & -4.50 & 0.63 \\
10 & 82 & F & RAY653L & +5.00 & 18.02 \\
11 & 75 & F & RAY653T & -1.00 & 0.94 \\
12 & 53 & M & RAY653T & +0.50 & 1.79 \\
13 & 62 & M & RAY653T & -3.00 & 0.55 \\
14 & 58 & M & RAY653T & -9.00 & 11.55 \\
\hline
\end{tabular}

Table 2. Patient preoperative and postoperative refraction.

\begin{tabular}{|c|c|c|}
\hline Eye & Preoperative Refraction & Postoperative Refraction \\
\hline 1 & $+3.25-0.50 \times 67$ & $-0.75-0.25 \times 66$ \\
2 & $+2.25-0.50 \times 158$ & $0.00-0.25 \times 180$ \\
3 & $+2.250 .00 \times 0$ & plano \\
4 & $+2.00-0.25 \times 74$ & $0.00-0.50 \times 15$ \\
5 & $+2.00-0.25 \times 30$ & $+0.25-0.25 \times 50$ \\
6 & $+1.50-0.25 \times 146$ & $-0.25-0.50 \times 100$ \\
7 & $-1.25-1.25 \times 85$ & $-0.25-0.25 \times 65$ \\
8 & $-4.25-0.75 \times 52$ & $+0.50-1.00 \times 75$ \\
9 & $+2.50-2.00 \times 165$ & $+0.75-0.50 \times 133$ \\
10 & $+1.25-1.00 \times 67$ & $+0.25-0.25 \times 55$ \\
11 & $+1.25-2.25 \times 95$ & $-0.50-0.50 \times 48$ \\
12 & $+1.00-0.50 \times 50$ & $-0.50-0.25 \times 171$ \\
13 & $-1.00-1.25 \times 124$ & $+1.75-0.75 \times 89$ \\
14 & $-3.25-3.75 \times 4$ & $-0.25-0.25 \times 90$ \\
\hline
\end{tabular}

Table 3. Targeted spherical equivalent and postoperative spherical equivalent by patient, in diopters (D).

\begin{tabular}{|c|c|c|c|}
\hline Eye & $\begin{array}{c}\text { Targeted spherical } \\
\text { equivalent }\end{array}$ & $\begin{array}{c}\text { Postoperative spherical } \\
\text { equivalent }\end{array}$ & $\begin{array}{c}\text { Difference between Targeted } \\
\text { and Postoperative SE }\end{array}$ \\
\hline 1 & -0.25 & -0.88 & -0.63 \\
2 & -0.10 & -0.13 & -0.03 \\
3 & -0.50 & 0.00 & +0.50 \\
4 & 0.00 & -0.25 & -0.25 \\
5 & 0.00 & 0.13 & +0.13 \\
6 & -0.50 & -0.50 & 0.00 \\
7 & 0.00 & -0.38 & -0.38 \\
8 & 0.00 & 0.00 & 0.00 \\
9 & -0.50 & -0.50 & 0.00 \\
10 & 0.00 & 0.13 & +0.13 \\
11 & -0.25 & -0.75 & -0.50 \\
12 & 0.00 & -0.63 & -0.63 \\
13 & 0.00 & +1.38 & +1.38 \\
14 & 0.00 & -0.38 & -0.38 \\
\hline
\end{tabular}


Table 4. The frequency of eyes whose postoperative spherical equivalent within range from the targeted spherical equivalent.

\begin{tabular}{|c|c|}
\hline Range from targeted SE & Number of eyes $(n=14)$ \\
\hline $\pm 0.25 \mathrm{D}$ & $7(50.0 \%)$ \\
$\pm 0.50 \mathrm{D}$ & $11(78.6 \%)$ \\
$\pm 0.75 \mathrm{D}$ & $13(92.9 \%)$ \\
$>1.00 \mathrm{D}$ & $1(7.1 \%)$ \\
\hline
\end{tabular}

Figure 1. Patient preoperative and postoperative uncorrected distance visual acuity (UDVA).

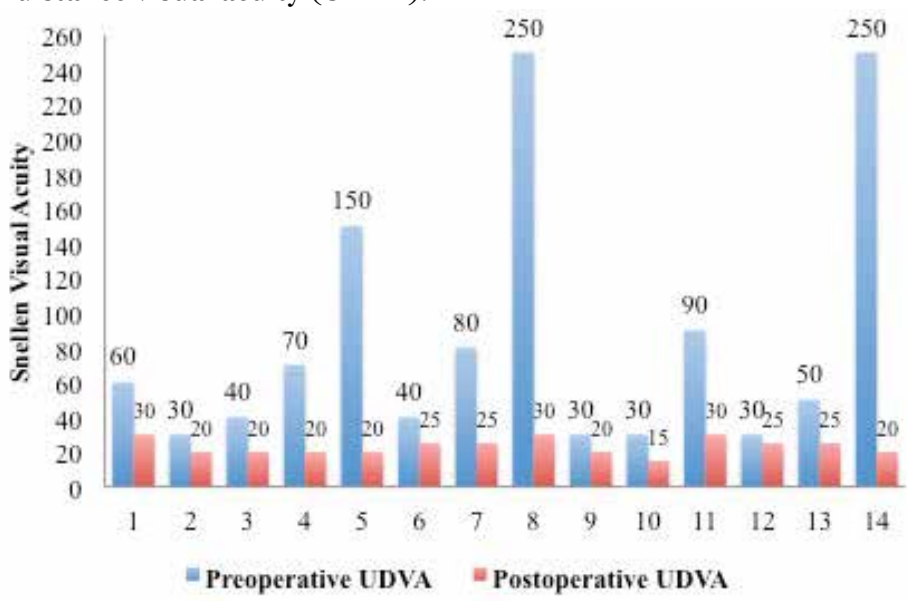

As shown in Table 2, the preoperative UDVA was $20 / 30$ in 4 eyes (28.6\%), 20/40 in 2 eyes (14.3\%), and was worse than $20 / 40$ in 8 eyes $(57.1 \%)$. The postoperative UDVA was $20 / 20$ or better in 7 eyes $(50.0 \%), 20 / 25$ in 4 eyes $(28.6 \%)$ and $20 / 30$ in 3 eyes $(21.4 \%)$. All patients had improved UDVA postoperatively. (Figure 1) The mean UDVA significantly improved from $0.50 \pm 0.33 \log$ MAR preoperatively to $0.06 \pm 0.09 \log$ MAR postoperatively, $p<.01$. There was no significant difference found when comparing the preoperative SCDVA $(0.05 \pm 0.12 \log$ MAR $)$ with the postoperative UDVA $(0.06 \pm 0.09 \log \mathrm{MAR}), p>0.05$.

No intraoperative or postoperative complications occurred in any case. There were no signs of pigment dispersion, Elschnig pearl formation, or interlenticular opacification observed during follow-up. Rotational stability and centration were excellent in all but one toric IOL case, which required a lens rotation 4 months postoperatively. After secondary surgery, the patient's vision stabilized at 20/20. Postoperatively, two eyes had a neodymium-doped yttrium aluminum garnet (Nd:YAG) laser capsulotomy for posterior capsular opacification. These capsulotomies were safely and successfully performed through both the Sulcoflex and the primary IOL.

One eye had a $+1.38 \mathrm{D}$ difference between the target SE and the postoperative SE. The refraction was +0.50 sphere one day postoperatively, however, by 6 months the refraction had gradually drifted more hyperopic. It is believed that the primary IOL had shifted in position due to capsular contraction, since a shift in the original $+30.0 \mathrm{D}$ lens would cause a greater change in refraction than the $-3.00 \mathrm{D}$ Sulcoflex lens. Upon further examination, it was found that the Sulcoflex IOL did have some rotation. However, the rotation does not have a great effect on the refraction, and is only an interesting coincidence.

\section{Discussion}

The findings of our study suggest that piggyback IOL implantation in the ciliary sulcus is a safe and viable option for the treatment of pseudophakic refractive error.

Similar to our findings, a study in the United Kingdom found a significant improvement in the spherical equivalent and uncorrected distance visual acuity after Sulcoflex implantation. ${ }^{15}$ In a sample of 15 eyes, all eyes had achieved a postoperative UDVA of 20/32 or better within 3 months with $10(67 \%)$ eyes achieving 20/20 or better. Falzon et al ${ }^{15}$ also found that $14(93 \%)$ eyes of were within $0.5 \mathrm{D}$ of the target refraction. Our study findings contribute to the current evidence regarding the efficacy of the Sulcoflex IOL to enhance refractive outcomes and reduce spectacle dependence for distance vision in pseudophakic eyes.

In our study, the postoperative spectacle corrected distance visual acuity was found to be comparable to postoperative distance visual acuity, which also suggests that the Sulcoflex IOL is an effective way to provide cataract patients freedom from spectacles. Given the treatment options available, some patients may choose to wear spectacles over receiving the secondary surgery. However, pseudophakic patients with anisometropia or significant residual ametropia would benefit from this procedure. Depending on the patient's demands for near and intermediate tasks, some patients may still require reading spectacles. The Sulcoflex IOL can be safely implanted after a long-term postoperative period, and as early as 3 months after primary cataract surgery if the refraction is deemed stable.

Although the Sulcoflex piggyback IOL appears to be a more predictable and safer option than the IOL exchange procedure, a past concern with the classic implantation of piggyback IOLs in the capsular bag was the possible formation of interlenticular opacification (ILO). ${ }^{16,17}$ ILO is opacification that occurs 
between the opposing lens surfaces and is a complication that can cause a significant decrease in vision as well as a possible hyperopic shift. ${ }^{17}$ Studies have demonstrated that implanting the piggyback lens in the ciliary sulcus instead of in the bag has been shown to reduce the risk of central optic touch. ${ }^{13}$ Since the development of lenses specifically designed for safe placement in the ciliary sulcus, the piggyback technique has regained attention for pseudophakic patients seeking to correct residual refractive errors. Although several different piggyback IOLs have been used, IOLs specifically designed for sulcus fixation, such as the Sulcoflex IOL are recommended. The round and smooth optic and haptic edges of the Sulcoflex lens reduce the risk of complications associated with single-piece IOLs with square, thick edges, such as pigmentary dispersion. ${ }^{4}$ There are also other alternative, commercially available piggyback IOL options designed for sulcus-placement, such as the Add-On (HumanOptics) and the 1st Add-On ( $1^{\text {st }}$ Q Deutschland GmbH \& Co.) IOLs.

The small sample size should be considered when interpreting the findings of this study. More studies are needed confirm the reliability and efficacy of this novel approach to correcting pseudophakic refractive error. A much larger

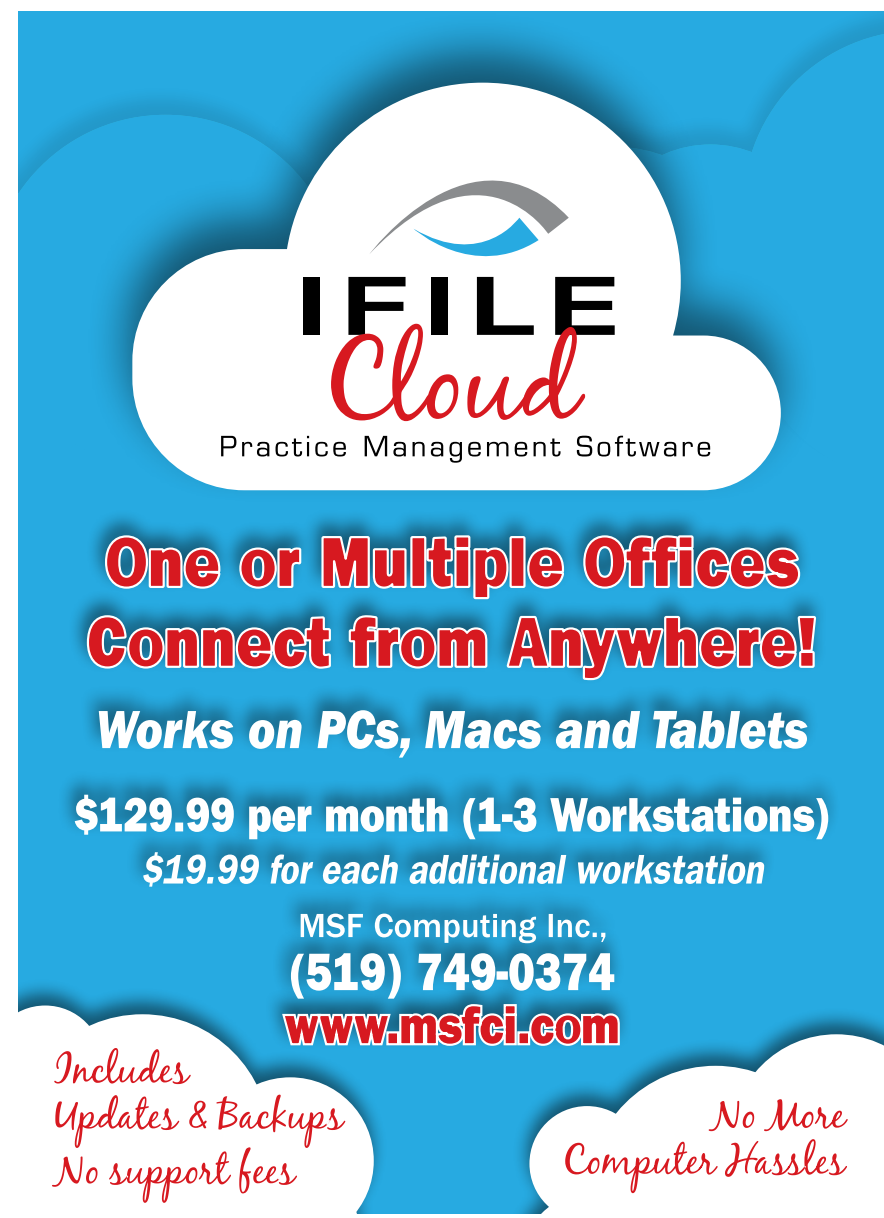

sample could increase the statistical power as well as adjust for factors such as sociodemographic characteristics, IOL type and power, as well as the degree of residua ametropia. Further studies are also necessary to better understand the long-term outcomes of Sulcoflex IOL implantation.

In conclusion, the findings of our study suggest that the Sulcoflex piggyback IOL is an effective treatment option for refractive errors or enhancement of postsurgical results, and can provide cataract patients freedom from spectacles many years after cataract surgery.

\section{References}

1. Amon M, Kahraman G. Enhancement of refractive results after intraocular lens implantation. European Ophthalmic Review 2011;5:59-61.

2. Habot-Wilner Z, Sachs D, Cahane M, et al. Refractive results with secondary piggyback implantation to correct pseudophakic refractive errors. J Cataract Refract Surg 2005; 31:2101-3.

3. Alio JL, Abdelghany AA, Fernandez-Buenaga R. Management of residual refractive error after cataract surgery. Curr Opin Opthalmol 2014;25:291-7.

4. Kahraman G, Amon M. New supplementary intraocular lens for refractive enhancement in pseudophakic patients. J Cataract Refract Surg 2010;36:1090-4.

5. Abdelghany AA, Alio JL. Surgical options for correction of refractive error following cataract surgery. Eye and Vision 2014;1.

6. El Awady HE, Ghanem AA. Secondary piggyback implantation versus IOL exchange for symptomatic pseudophakic residual ametropia. Graefes Arch Clin Exp Ophthalmol 2013; 251:1861-6.

7. Jivrajka RV, Shammas MC, Shammas HJ. Improving the second-eye refractive error in patients undergoing bilateral sequential cataract surgery. Ophthalmology 2012; 119:1097-101.

8. Rubenstein JB. "Piggyback IOLs for residual refractive error after cataract surgery," Cataract Refractive Surgery Today, August 2012, pages 28-30.

9. Wallace III RB, Trattler WB, Waltz KL, et al. "Piggyback IOLs: common indications, preferred IOLs, and surgical techniques," Cataract Refractive Surgery Today, April 2011, 27-29.

10. McIntyre JS, Werner L, Fuller SR, et al. Assessment of a single-piece hydrophilic acrylic IOL for piggyback sulcus fixation in pseudophakic cadaver eye. J Cataract Refract Surg 2012;38:155-62.

11. Clare G, Bloom P. Bilateral ciliary sulcus implantation of secondary piggyback multifocal intraocular lenses. J Cataract Refract Surg 2007;33:320-2.

12. Alfonso JF, Fernandez-Vega L, Baamonde MB. Secondary diffractive bifocal piggyback intraocular lens implantation. J Cataract Refract Surg 2006;32:1938-43.

13. Baumeister M, Kohnen T. Scheimpflug measurement of intraocular lens position after piggyback implantation of foldable intraocular lenses in eyes with high hyperopia. J Cataract Refract Surg 2006;32:2098-104

14. "Product Portfolio: Sulcoflex Pseudophakic Supplementary IOLs.” Rayner. Rayner Intraocular Lenses Unlimited, Oct. 2013. Web. 7 Apr. 2015.10.

15. Falzon K, Stewart OG. Correction of undesirable pseudophakic refractive error with Sulcoflex intraocular lens. J Refract Surg 2012;28(9):614-619.

16. Werner L, Apple DJ, Pandey SK, et al. Analysis of elements of interlenticular opacification. Am J Ophthalmol 2002;133:320-6.

17. Werner L, Shugar JK, Apple DJ, et al. Opacification of piggyback IOLs associated with an amorphous material attached to interlenticular surfaces. J Cataract Refract Surg 2000;26:1612-9. 